Proceedings

\title{
Apta- and Immuno-Sensors Performance Optimization: A Comparative Study of Surface Functionalization Techniques ${ }^{+}$
}

\author{
George Tsekenis ${ }^{1}$, Valentina Vanikioti ${ }^{2}$, Konstantinos Kordatos ${ }^{2}$ and Ioanna Zergioti ${ }^{2, *}$ \\ 1 Biomedical Research Foundation of the Academy of Athens, 11527 Athens, Greece; \\ gtsekenis@bioacademy.gr \\ 2 School of Chemical Engineering, National Technical University of Athens, 15780 Athens, Greece; \\ valentinavanikioti@gmail.com (V.V.); kordatos@central.ntua.gr (K.K.) \\ 3 School of Applied Mathematical and Physical Sciences, National Technical University of Athens, 15780 \\ Athens, Greece \\ * Correspondence: zergioti@central.ntua.gr; Tel.: +30-2107-723-345 \\ † Presented at the Eurosensors 2018 Conference, Graz, Austria, 9-12 September 2018. \\ Published: 11 December 2018
}

\begin{abstract}
Surface bio-functionalization plays a critical role in the performance of a biosensor and numerous techniques for the enhancement of a biosensor's surface coverage with oriented capture biomolecules have been developed with the ultimate goal of optimizing a sensor's performance in terms of its sensitivity and linear response over a wide dynamic range. Herein, highlights of a comparative assessment into the most promising approaches to achieve this goal are being presented. For aptamer-modified surfaces, polyamidoamine (PAMAM) dendrimers and polysaccharide networks were employed with the obtained results clearly indicating that a much denser surface coverage with aptamers can be achieved with the use of the latter. For the functionalization of surfaces with antibodies, the orientation and density of immobilized antibodies onto recombinant protein A/G- or boronic acid-modified substrates were compared, with the former leading not only to increased antibody loading but also with such an orientation that permits enhanced antigen binding. The conclusions reached can be used as a starting point for the customization of sensor functionalization in a plethora of clinical, environmental and even foodindustry-related biosensing platforms.
\end{abstract}

Keywords: immunosensor; aptasensor; surface functionalization enhancement

\section{Introduction}

One critical consideration during the development of a biosensor is the immobilization of capture biomolecules onto a solid support with a sufficient surface density, and an orientation that maximizes their target analyte capture potential. These parameters play a critical role in the performance of a biosensor as they will influence its sensitivity and reproducibility as well as its response over a wide dynamic range. A significant amount of effort has been put towards improving these parameters for biosensors that rely on the use of antibodies or aptamers, two of the most commonly employed types of capture biomolecules in biodiagnostic or analytical assays [1]. Nevertheless, the majority of the published work focuses on the optimization of a single protocol specific to the biosensor under development. Review papers, on the other hand, are useful in presenting the techniques developed by various research groups to address these issues, however they do not permit a truly valid comparison to be made. There exists, therefore, the need for a comparative analysis of different surface biofunctionalization techniques by employing the same 
capture biomolecule, with the ultimate goal being the generation of a set of generally applicable guidelines rather than establishing the optimum protocol for a specific application.

Herein, a small subset of the results obtained following the bio-functionalization of silicon-based substrates with aptamers and antibodies are presented. The most promising techniques for their orientation-specific immobilization onto silanized surfaces were employed with the scope of increasing their surface loading. For aptamers, two different approaches, polyamidoamine (PAMAM) dendrimers and polysaccharides, were followed and their efficiency was compared in relation to the length of the aptameric sequences [2]. For antibodies, the silanized silicon nitride surfaces were functionalized with either recombinant protein $A / G$ or aminophenyl boronic acid (APBA), both of which have shown potential in achieving the oriented immobilization of antibodies through the specific interaction either directly with or through polysaccharides in the constant region (Fc) of the antibody [3]. A more extensive discussion of the conclusions that were reached will be presented in articles to be published in the future.

\section{Materials and Methods}

The aptamer sequence against OTA (Texas Red conjugated) [4] was synthesized and purchased from Aldrich Chemicals, while the Donkey anti-Rat IgG Cy5 conjugated (2ary antibody) was purchased from Jackson Immunoresearch and the rat-raised Anti-CD24 PE conjugated (1ary antibody) from Thermo Fisher Scientific. Recombinant protein A/G and a Pierce ${ }^{\mathrm{TM}}$ Antibody Cleanup Kit were also purchased from Thermo Fisher Scientific. All other reagents were purchased from Aldrich Chemicals. All solutions were prepared with deionized (DI) water (18 $\mathrm{M} \Omega / \mathrm{cm}$ resistivity, Millipore MilliQ).

\subsection{Surface Silanization and (Bio)-Chemical Modifications}

For the functionalization of the silicon nitride $\left(\mathrm{Si}_{3} \mathrm{~N}_{4}\right)$ surfaces (3-glycidyloxypropyl)triethoxysilane (GOPTS) was used, according to a previously published protocol [5]. Prior to their silanization, the surfaces were cleaned by sonication in acetone and isopropanol and immersed in a solution of $1 \mathrm{M} \mathrm{NaOH}$ for $1 \mathrm{~h}$, rinsed with DI water and dried with nitrogen gas.

\subsubsection{Aptamer-Based Sensors}

The surfaces were exposed to different concentration of PAMAM dendrimers dissolved in $1 \%$ methanol overnight and were subsequently rinsed with methanol and dried with nitrogen gas. The surfaces were then treated for 2 hours with a solution of $2.5 \%$ glutaraldehyde in PBS $1 \times \mathrm{pH} 7.4$ and thoroughly washed with the same buffer. Alternatively, for the modification of the surfaces with polysaccharides the silanized substrates were exposed overnight to solutions of either $0.5 \%(w / v)$ chitosan in acetic acid or $0.5 \%(w / v)$ hyaluronic acid in DI water and were subsequently rinsed with DI water and dried with nitrogen gas. Chitosan-functionalized surfaces were then incubated with 2.5 $\%(v / v)$ glutaraldehyde while hyaluronic acid surface were NHS-terminated by the addition of $0.4 \mathrm{M}$ EDC/ 0.1 M NHS in 0.1 M MES buffer $\mathrm{pH} 4.5$ for 15 minutes.

\subsubsection{Antibody-Based Sensors}

Following silanization the surfaces were incubated overnight with either $100 \mu \mathrm{g} / \mathrm{mL}$ recombinant protein A/G in $10 \mathrm{mM}$ Carbonate Buffer $\mathrm{pH} 9.2$ or 3\% APBA in DI water. Protein A/G-modified surfaces were subsequently washed with the used buffer while the APBA-functionalized surfaces were first rinsed with ethanol and then water.

\subsection{Aptamer and Antibody Immobilization}

Both aptamers as well antibodies were drop-casted onto the functionalized surfaces. Prior to the application of antibodies onto the chemically-functionalized surfaces, BSA that acts as a stabilizer was removed with the use of a Pierce ${ }^{\mathrm{TM}}$ Antibody Clean-up Kit according to the manufacturer's instructions. The aptamers were prepared in $10 \mathrm{mM}$ phosphate buffer $\mathrm{pH} 7.4$, containing $10 \mathrm{mM} \mathrm{KCl}$, 
$5.0 \mathrm{mM} \mathrm{MgCl} 2$ and were left to immobilize onto the surfaces overnight at $4{ }^{\circ} \mathrm{C}$ prior to being rinsed with use of the same buffer. Secondary antibody solutions were prepared in either PBS 1x pH 7.4 (for protein $\mathrm{A} / \mathrm{G}$ treated surfaces) or $10 \mathrm{mM}$ Carbonate buffer $\mathrm{pH} 9.2$ (for APBA and GOPTSfunctionalized surfaces) and were incubated with the surfaces overnight at $4{ }^{\circ} \mathrm{C}$ prior to being rinsed with the used buffers respectively. The bioreactivity of the secondary antibodies was investigated through incubation with solutions of different concentrations of the 1ary antibody in PBS 1x pH 7.4 (with $0.5 \%$ Tween-20 and 1\% BSA). Prior to their exposure to their antigen, the antibody-modified surfaces were blocked as follows: GOPTS and protein A/G-modified surfaces were incubated with a solution of $10 \mathrm{mM}$ ethanolamine in $10 \mathrm{mM}$ Carbonate buffer $\mathrm{pH} 9.2$ (for $30 \mathrm{~min}$, rinsed with the same buffer and then exposed for a further 30 min to PBS 1x pH 7.4 (with 0.5\% Tween-20 and 1\% BSA). APBA surfaces were blocked with a solution of $0.25 \%(w / v)$ dextran (MW 6000) in PBS $1 \times$ pH 7.4 for 30 minutes.

\subsection{Visualization of the Immobilized and Captured Biomolecules}

Finally, the surfaces were extensively rinsed to remove any unbound analyte. A Leica fluorescent microscope was used to acquire fluorescent images. In all cases, the recorded fluorescence was quantified with the use of the ImageJ software (following subtraction of the background). Quantification of the fluorescence intensity was performed with the use of the ImageJ software (1.8.0_112 version).

\section{Results and Discussion}

\subsection{Apta-Sensors}

A thorough investigation into the effect different concentrations the PAMAM dendrimers of four generations (G0 to G3) have on the amount of aptamer immobilized onto the surfaces was carried out. The results indicate that considerably more aptamer is immobilized onto the 3D dendrimer structures in comparison to flat surfaces (Figure 1).

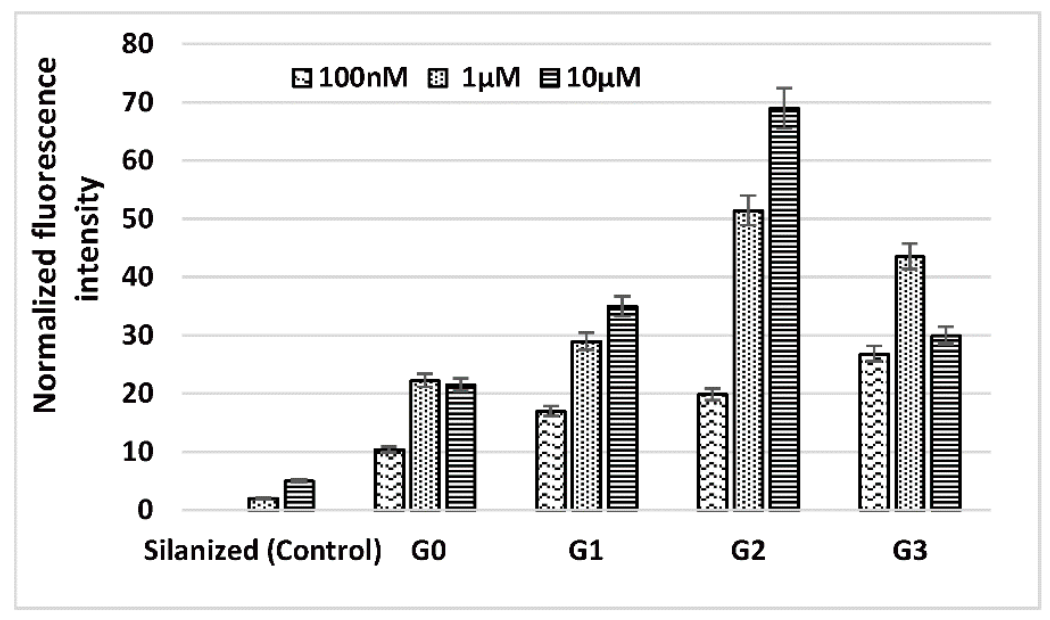

Figure 1. Recorded fluorescence intensity from three different concentrations of amino-modified aptamer against Ochratoxin A (tagged with Texas Red) immobilized onto silanized surfaces that have been functionalized with four generations of PAMAM dendrimers at a concentration of $1 \% v / v$ in methanol.

An increased number of immobilized aptamers can be observed as the number of functional groups present on the surfaces increases with higher generations of PAMAM (G0 up to G2). Despite the enhanced surface coverage achieved with PAMAM dendrimers, an even denser network of aptamers on the surfaces can be achieved with the use of chitosan as the results in Figure 2 illustrate. 


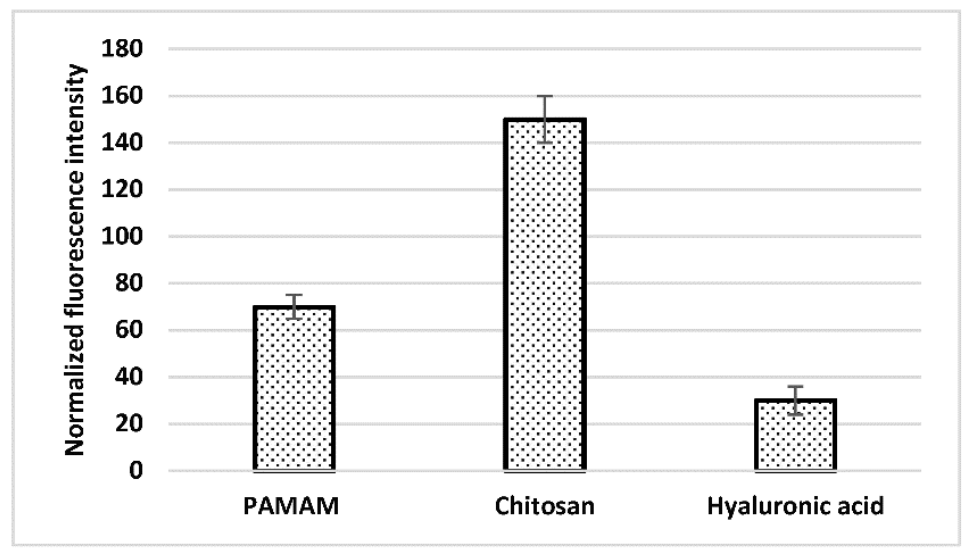

Figure 2. Recorded fluorescence intensity from $1 \mu \mathrm{M}$ aptamer against Ochratoxin A (tagged with Texas Red) immobilized onto silanized surfaces that have been functionalized with 1\% PAMAM G2, $0.5 \%$ chitosan or $0.5 \%$ hyaluronic acid.

\subsection{Immunosensors}

Analysis of the obtained fluorescence intensity results reveals that significantly higher amounts of antibody get immobilized onto the surfaces with the use of protein A/G in comparison to APBAmodified surfaces, whose loading capacity is comparable with that of unmodified, silanized surfaces (Figure 3).

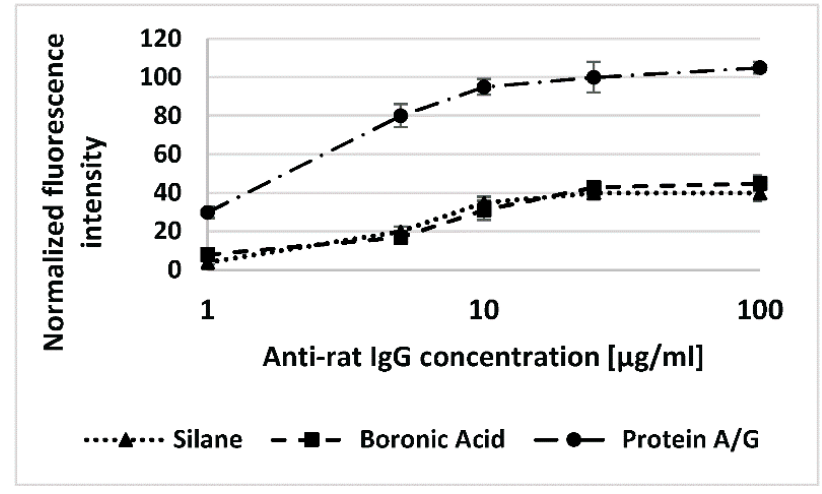

Figure 3. Antibody loading efficiency for 5 different concentrations of Cy5-labelled Anti-rat IgG immobilized onto aminophenyl boronic acid and proteinA/G-functionalized surfaces in comparison to only surfaces.

Nevertheless, the orienation-specific immobilization of antibodies onto APBA-fucntionalized surfaces does contribute to their improved binding capacity for their antigen as the fluorescence images in Figure 4 reveal.
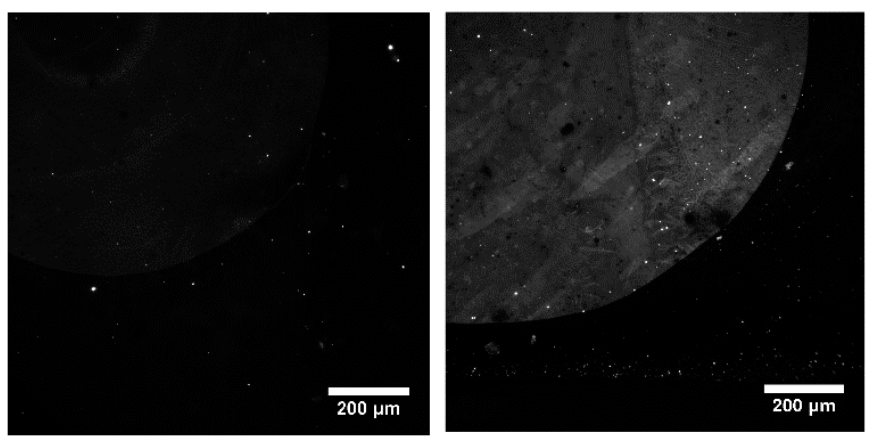

Figure 4. Fluorescence intensity from bound 1ary antibody onto $10 \mu \mathrm{g} / \mathrm{mL}$ immobilized 2ary antibody onto silanized surfaces (left) and surfaces treated with 3\% APBA (right). 
Author Contributions: G.T. conceived and designed all of the experiments and performed the experiments on the immunosensors; V.V. performed the experiments on aptasensors; K.K. supervised V.V. in her Master's thesis and offered his guidance; I.Z. had the overview of the whole research group and offered her guidance and support.

Acknowledgments: The work was supported by the EU-funded FP7 project ICT-BIOFOS (Contract No. 61528) and the EU-funded H2020 project ICT-BIOCDx (Contract No. 732309).

Conflicts of Interest: The authors declare no conflict of interest

\section{References}

1. Welch, N.G.; Scoble, J.A.; Muir, B.W.; Pigram, P.J. Orientation and characterization of immobilized antibodies for improved immunoassays (review). Biointerphases 2017, 12, 02D301. doi:10.1116/1.4978435

2. Singh, R.P.; Tiwari, A.; Choi, J.W.; Pandey, A.C. Smart nanomaterials for biosensors, biochips and molecular bioelectronics. In Smart Nanomaterials for Sensor Applications, 2nd ed.; Li, S., Ge, Y., Li, H., Eds.; Bentham Science Publishers: Beijing, China, 2012; pp. 3-41.

3. Trilling, A.K.; Beekwilder, J.; Zuilhof, H. Antibody orientation on biosensor surfaces: A minireview. Analyst 2013, 138, 1619-1627. doi:10.1039/c2an36787d.

4. Cruz-Aguado, J.A.; Penner, G.; Determination of Ochratoxin A with a DNA Aptamer. J. Agric. Food Chem. 2008, 56, 10456-10461. doi:10.1021/jf801957h.

5. Tsekenis, G.; Chatzipetrou, M.; Tanner, J.; Chatzandroulis, S.; Thanos, D.; Tsoukalas, D.; Zergioti, I. Surface functionalization studies and direct laser printing of oligonucleotides toward the fabrication of a micromembrane DNA capacitive biosensor. Sens. Actuators B Chem. 2012, 175, 123-131. doi:10.1016/j.snb.2012.01.005

(C) 2018 by the authors. Licensee MDPI, Basel, Switzerland. This article is an open access article distributed under the terms and conditions of the Creative Commons Attribution (CC BY) license (http://creativecommons.org/licenses/by/4.0/). 